\title{
Docking Studies, Synthesis and Evaluation of Anticancer Activity of 4H-Chromene Derivatives
}

\author{
Piyush Kumar ${ }^{1}$, Pinki Rawat ${ }^{2, *}$ \\ 1'Department of Pharmacy, Integral University, Lucknow, Uttar Pradesh, INDIA. \\ ${ }^{2}$ Department of Pharmacy, Maharana Pratap College of Pharmacy, Kanpur, Uttar Pradesh, INDIA.
}

\begin{abstract}
Aim: The present study involves to design, synthesis and evaluate the anticancer activity of $4 \mathrm{H}$-chromene derivatives (PKB 1-10) on human breast cancer MCF-7 cell line. Materials and Methods: $4 \mathrm{H}$-chromene derivatives (PKB 1-10) were designed by docking, in silico ADME and predicted toxicity studies. These designed compounds were then synthesized by acid catalyzed Michael Addition of phenols to benzylidene oxobutanoates. These compounds were characterized by ${ }^{1} \mathrm{H}$ NMR, ${ }^{13} \mathrm{C}$ NMR, FTIR and melting point. Then all synthesized compounds were tested for anticancer activity on MCF-7 cell line. Results: Compounds PKB-4 and PKB-10 showed better docking scores than standard drug, Adriamycin. In silico ADME and toxicity studies were also found significant for most of the compounds. The majority of the compounds displayed promising to potent anticancer activity on MCF-7 cell line. Conclusion: It may be concluded that most of the compounds showed significant docking and in silico ADME and toxicity profiles. Compounds have excellent anticancer potential and could be considered as novel anticancer agents for more investigation.
\end{abstract}

Key words: Anticancer, 4H-chromenes, Docking, MCF-7 cell line, Toxicity.

\section{INTRODUCTION}

Cancer is one of the leading causes of death in the world among all chronic diseases. ${ }^{1}$ Breast cancer is the most prevalent form of cancer diagnosed in women worldwide and affecting 2.1 million women each year. According to 2018 report, the estimated number of female died due to breast cancer was 6.2 million. It is accounting approximately $15 \%$ of all cancer deaths among women. ${ }^{2}$ There are many difficulties in the treatment of cancer, but the most concerning are drug resistance, toxicity and low specificity. ${ }^{3}$ Therefore, there has been increscent interest in the field of cancer chemotherapy by discovery and development of novel agents with high efficacy, low toxicity and minimum side effects. ${ }^{4}$ Due to the less selectivity of suitable drugs, drug resistance and complex mechanisms, the current drug treatment of breast cancer seem to be challenging. ${ }^{5}$ Therefore, the development of potent, competent and having less adverse effect anticancer agents over the synthesis of new molecules is significant in breast cancer research.

4H-Chromenes are important heterocyclic pharmacophores with a benzene ring fused to a pyran nucleus. They are found in natural and synthetic compounds and have recently attracted much more interest due to their useful biological and pharmacological properties. A wide range of new chromene-based natural products were reported during the last two decades. ${ }^{6}$ These heterocyclic natural and synthetic benzopyran derivatives possess important biological properties such as anticancer, ${ }^{7}$ anti-coagulant, ${ }^{8}$ antimicrobial, ${ }^{9}$ anti-proliferative, ${ }^{10}$ antioxidant, ${ }^{11}$ antifungal,$^{12}$ antiestrogenic, ${ }^{13}$ antiviral, ${ }^{14}$ antitubercular, ${ }^{15}$ anti-inflammatory ${ }^{16}$ and anticonvulsant ${ }^{17}$ activities. During recent years, several researchers developed
Submission Date: 20-06-2020; Revision Date: 09-09-2020; Accepted Date: 18-12-2020

DOI: 10.5530/ijper.55.1.29 Correspondence: Dr. Pinki Rawat

Associate Professor, Department of Pharmacy, Maharana Pratap College of Pharmacy, Kanpur-209217, Uttar Pradesh, INDIA. Phone: +919140815497 E-mail: pnkrawat@yahoo. co.in

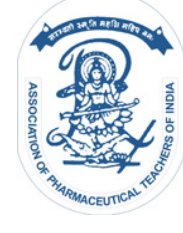

www.ijper.org 
different 4H-chromene derivatives with anti-breast cancer activity through the introduction of various heterocyclic scaffolds (Figure 1).

Recently, high-throughput screening of drug libraries results in the identification of some new $4 \mathrm{H}$-chromene compounds that exhibited cytotoxic activity against breast cancer cell lines MCF-7 and MBD-MB-231. MCF-7 breast cancer cells are estrogen (E2) sensitive cells and depend on E2 in order to proliferate. They express high levels of $\mathrm{ER} \alpha$ transcripts but low levels of ER $\beta$. They are used pervasively in research for ER-positive breast cancer cell experiments and development of anti-estrogen drug. MCF-7 cells are well-suited for antihormone therapy studies since they are readily cultured and retain ER expression when they were treated with such targeted-therapy. ${ }^{18}$

The Cytochromes P450 (CYPs) constitute the major enzyme family efficient of catalyzing the oxidative biotransformation of most drugs and therefore of particular relevance for clinical pharmacology. ${ }^{19}$ Overexpression of P-glycoprotein leads to multidrug resistance in the ovarian cancer cell line. ${ }^{20}$ Accordingly, in continuation of our research program to find antibreast cancer agents and considering the importance of $4 \mathrm{H}$-chromenes as anti-breast cancer agents, we have synthesized a series of $4 \mathrm{H}$-Chromene derivatives.

Therefore, we hereby report the design, synthesis and anticancer property of $4 \mathrm{H}$-chromene derivatives (PKB 1-10). These compounds were screened for their antibreast cancer properties on the MCF-7 cell line.

\section{MATERIALS AND METHODS}

All chemicals used were procured commercially from Spectrochem and Avra Synthesis Pvt. Ltd. Solvents were dried and distilled according to conventional procedures. Reactions were carried out under nitrogen $\left(\mathrm{N}_{2}\right)$

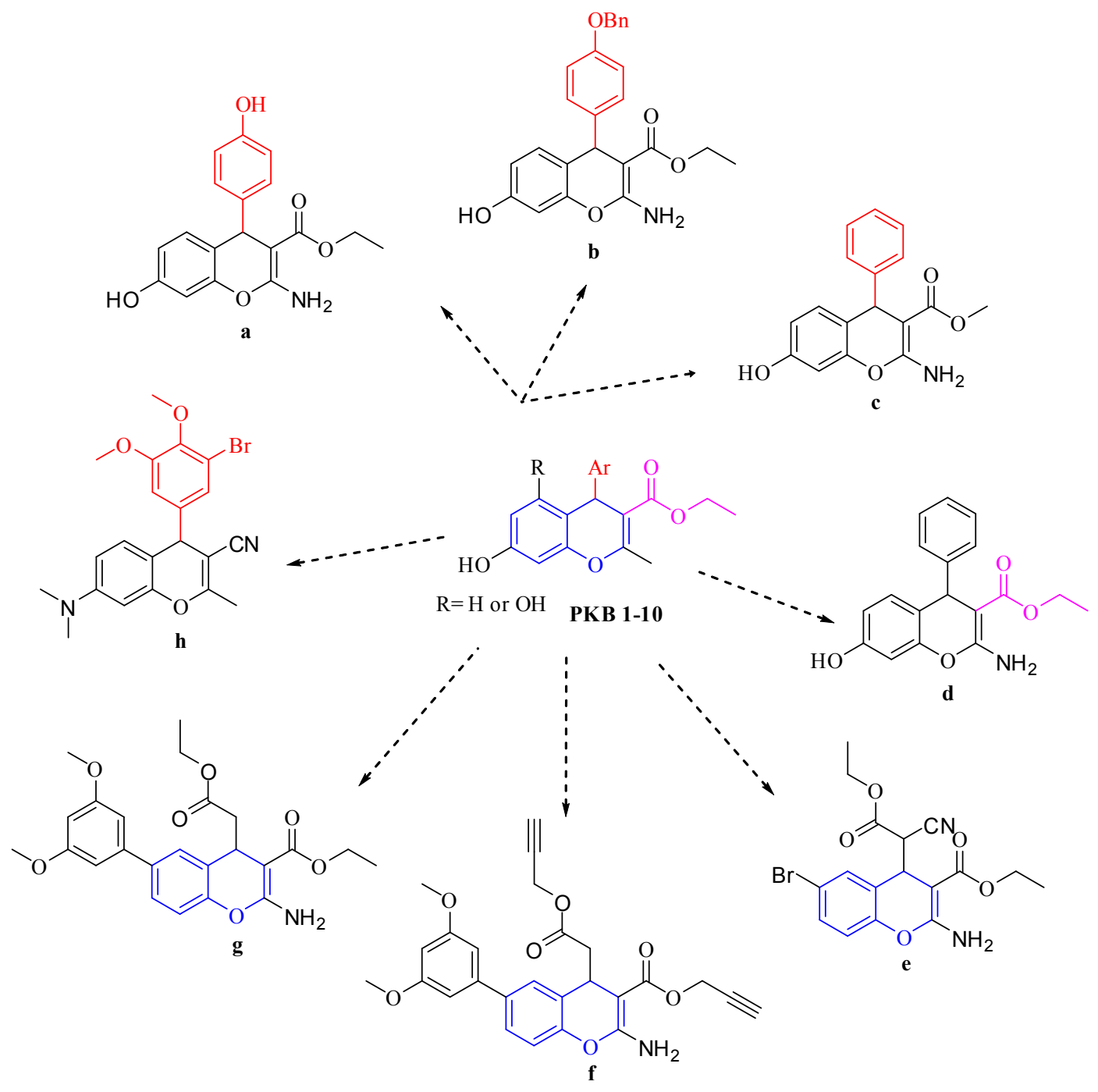

Figure 1: Pharmacophoric pattern of anticancer drugs (a-h) and model compounds (PKB 1-10). 
atmosphere. The progress of the reaction was checked by Thin Layer Chromatography (TLC). Melting points were measured in open glass capillaries. NMR spectra were carried out on the JEOL ECX-400 spectrometer in DMSO- $d^{6}$ and $\mathrm{CDCl}_{3}$ at $400 \mathrm{MHz}$ for ${ }^{1} \mathrm{H}$ and 100 $\mathrm{MHz}$ for ${ }^{13} \mathrm{C}$. Chemical shifts $(\delta)$ were determined in $\delta$ parts per million (ppm) relative to TMS. The mass spectra of samples were carried out on Waters Q-Tof Premier-HAB213 spectrometer. The FTIR spectra $(\mathrm{KBr})$ of samples were carried out on a PerkinElmer Spectrum Version 10.03.06 spectrophotometer.

\section{RESULTS AND DISCUSSION}

\section{Molecular Docking}

The molecular docking studies of compounds (PKB 1-10) was performed by Autodock 1.4.6 software on estrogen receptor (PDB: 2POG) for anti-breast cancer screening (Table 1). The docking score of compounds was ranging between -10.26 to -7.10 . The compound PKB-10 having 5-bromothiophen moiety (-10.26) showed the highest and better docking scores than standard drug, Adriamycin (-9.90). Other compound<smiles>[R]c1cc(O)cc2c1C([Al])C(C(=O)OCC)=C(C)O2</smiles>

PKB 1-10

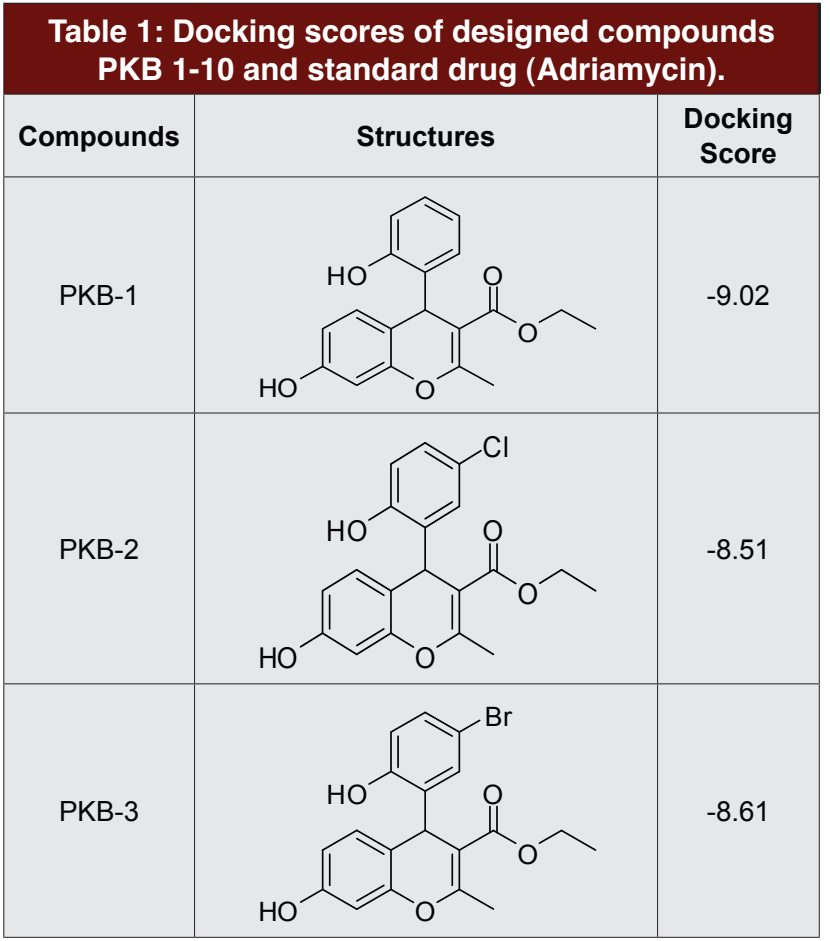

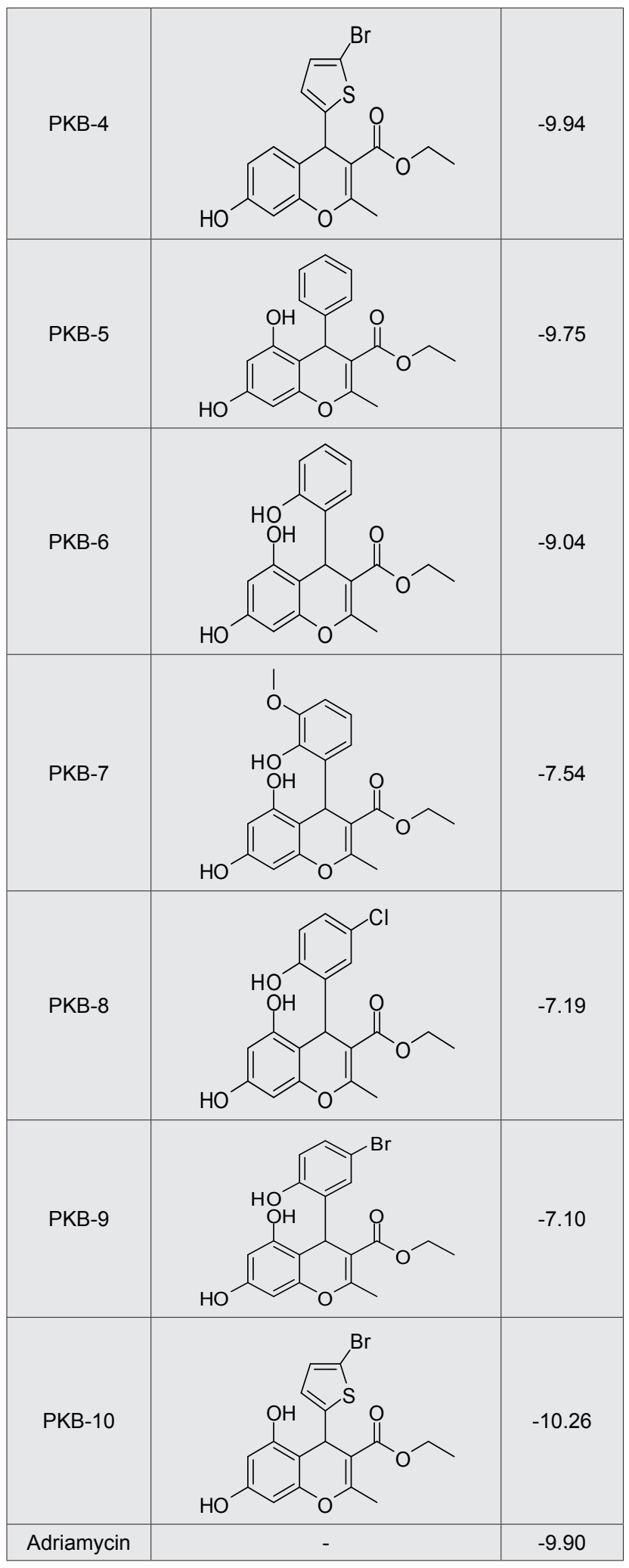

PKB-4 having 5-bromothiophen moiety (-9.94) with one hydroxyl group also possessed great result. Therefore, it can be postulated that compound with 5 -bromothiophen moiety displayed best results among the series. Compounds PKB-1 (-9.02), PKB-5 (-9.75) and PKB-6 (-9.04) also exhibited promising score. The 
compound PKB-7 (-7.54) possess 5-methoxyphenyl group yielded less score as compared to other compounds having only hydroxyl group. Thus, presence of methoxy group yielded less activity. The results showed that the presence of electronegative groups (chlorine and bromine) were responsible for a significant docking score. The binding pose of best docked compound PKB-10 with alpha ligandbinding domain of the estrogen receptor was shown in Figure 2. The -O- of hydroxyl group in highest docking scored compound PKB-10 involved in hydrogen bonding interaction with LEU346 via $-\mathrm{H}$ of $-\mathrm{NH}$. The visualization pose of the ligand WST (3AS,4R,9BR)-4-(4-Hydroxyphenyl)-1,2,3,3a,4,9b-

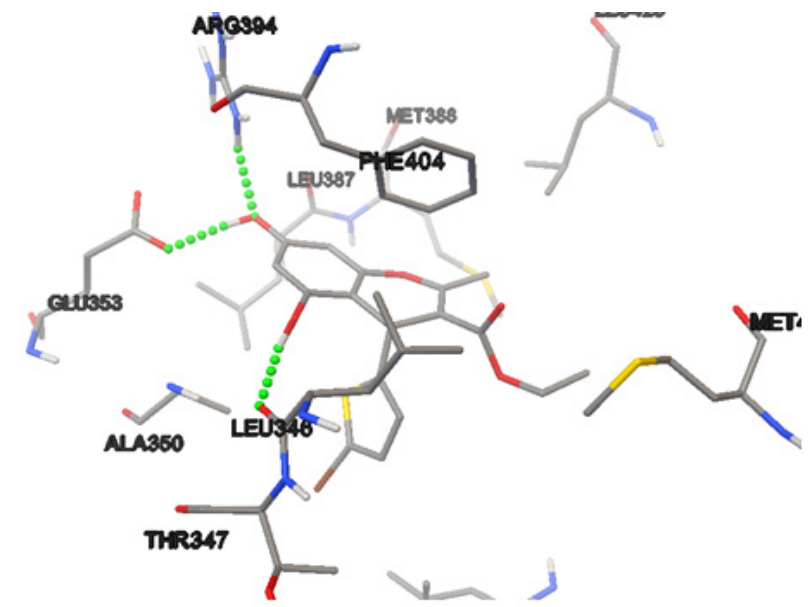

Figure 2: Docked conformation of PKB-10 showing important amino acid residues of the alpha ligand-binding domain of the estrogen receptor. $\mathrm{H}$-bonds are displayed in green dotted line. hexahydrocyclopenta[c]chromen-9-ol) with ER $\alpha$ binding pocket of the estrogen receptor was shown Figure 3.

\section{Predicted ADME Studies}

All designed compounds (PKB 1-10) showed high gastrointestinal absorption (GI). Compounds PKB1, PKB-2 and PKB-5 showed BBB permeability. Compounds PKB-2, PKB-6 and PKB-7, displayed inhibition to P-glycoprotein. All compounds showed inhibition to Cytochrome P 450 isomers (CYP1A2). Compounds PKB-1, PKB-5, PKB-6, PKB-7, PKB-8 and PKB-9 possessed inhibition for CYP2D6. All compounds followed drug-likeness prediction depending on the selected Ghose, Lipinski and Veber rule. All compounds displayed excellent bioavailability score. It could be seen that the majority of compounds among the series possessed better pharmacokinetic properties (Table 2). The ADME results of compounds (PKB 1-10)

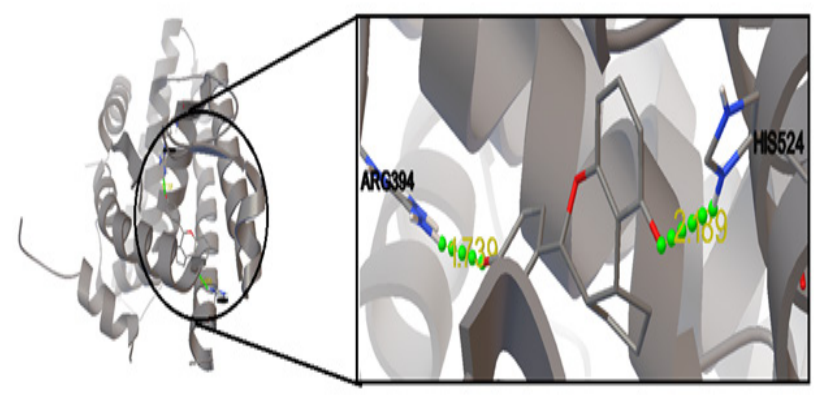

Figure 3: Autodock-predicted visualization pose of the ligand WST with ERa binding pocket of the estrogen receptor.

Table 2: Pharmacokinetic studies (ADME) of compounds (PKB 1-10).

\begin{tabular}{|c|c|c|c|c|c|c|c|c|c|c|}
\hline \multirow[b]{2}{*}{ 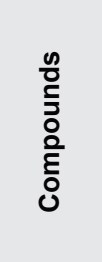 } & \multicolumn{6}{|c|}{ Pharmacokinetics } & \multicolumn{4}{|c|}{ Drug-likeness } \\
\hline & 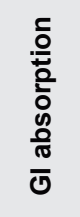 & 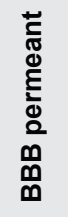 & $\begin{array}{l}0 \\
\text { 움 } \\
0\end{array}$ & $\frac{\frac{\pi}{d}}{\frac{1}{d}}$ & $\begin{array}{l}\text { م } \\
\text { ก } \\
\text { o }\end{array}$ & 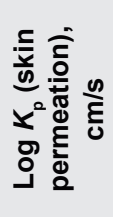 & $\begin{array}{l}\frac{\bar{x}}{0} \\
. \frac{2}{3} \\
.0\end{array}$ & 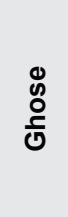 & $\begin{array}{l}\frac{1}{ \pm} \\
\stackrel{\circ}{\circ}\end{array}$ & 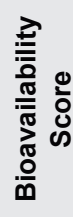 \\
\hline PKB-1 & High & Yes & No & Yes & Yes & -5.89 & Yes & Yes & Yes & 0.56 \\
\hline PKB-2 & High & Yes & Yes & Yes & No & -5.65 & Yes & Yes & Yes & 0.56 \\
\hline PKB-3 & High & No & No & Yes & No & -5.88 & Yes & Yes & Yes & 0.56 \\
\hline PKB-4 & High & No & No & Yes & No & -5.54 & Yes & Yes & Yes & 0.56 \\
\hline PKB-5 & High & Yes & No & Yes & Yes & -5.89 & Yes & Yes & Yes & 0.56 \\
\hline PKB-6 & High & No & Yes & Yes & Yes & -6.24 & Yes & Yes & Yes & 0.56 \\
\hline PKB-7 & High & No & Yes & Yes & Yes & -6.45 & Yes & Yes & Yes & 0.56 \\
\hline PKB-8 & High & No & No & Yes & Yes & -6.24 & Yes & Yes & Yes & 0.56 \\
\hline PKB-9 & High & No & No & Yes & Yes & -6.24 & Yes & Yes & Yes & 0.56 \\
\hline PKB-10 & High & No & No & Yes & No & -5.88 & Yes & Yes & Yes & 0.56 \\
\hline
\end{tabular}

P-gp: P-glycoprotein; GI: Gastro Intestinal; BBB: Blood Brain Barrier; CYP1A2: Cytochrome P450 family 1 subfamily A member 2 (PDB: 2HI4); CYP2D6: Cytochrome P450 family 2 subfamily $D$ member 6 (PDB: 5 TFT). 
were compared with adriamycin, an anti-breast cancer drug. Adriamycin displayed low oral bioavailability ${ }^{21}$ and poor penetration into the brain. ${ }^{22}$ The efflux of the adriamycin was mediated by P-glycoprotein ${ }^{23}$ and it competitively inhibits cytochrome P450 enzymes in human liver microsomes. ${ }^{24}$

\section{Predicted Toxicity Studies}

All compounds predicted to be non-mutagenic and noncarcinogenic except for compound PKB-7 which was found to be mutagenic. All compounds had significant drug score. None of the compounds showed positive DL values. Compound PKB-6 displayed better solubility than other compounds. High cLogP (CLP) values are an estimation of low hydrophilicity and therefore cause low absorption or permeation. Compounds PKB-1, PKB-5, PKB-6 and PKB-7, displayed significant cLogP values (Table 3). The toxicity results of compounds (PKB 1-10) were compared with adriamycin. Despite the clinical effectiveness of adriamycin, its use is limited by off-target adverse effects, particularly dose-related cardiotoxicity, systemic toxicities and renal toxicity, which involves the free radical formation and tissue damage. ${ }^{22}$

\section{Chemistry}

The $4 \mathrm{H}$ chromene derivatives (PKB 1-10) were synthesized by following known procedures of literature. ${ }^{25}$ The first step involved the synthesis of substituted ethyl benzylidiene oxobutanoates 3 by the reaction between substituted aromatic aldehydes 1 and ethyl acetoacetate 2 in DMSO at $80^{\circ} \mathrm{C}$ using base L-proline. The synthesis of final compounds (PKB 1-10) was accomplished by trifluoroacetic acid (TFA) catalyzed Michael addition of phenols 4 to different substituted ethyl benzylidiene oxobutanoates 3. In all cases, the targeted final $4 \mathrm{H}$ chromene derivatives (PKB 1-10) were obtained in good yield (Scheme 1).

Compounds (PKB 1-10) displayed a characteristic broad absorption band of the hydroxyl group $(-\mathrm{OH}$, stretch) at $3333-3512 \mathrm{~cm}^{-1}$. In ${ }^{1} \mathrm{H} \mathrm{NMR}$, the $-\mathrm{CH}_{3}$ of

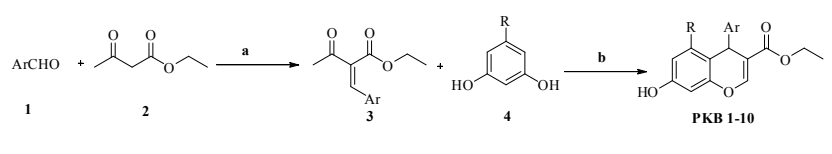

$\mathrm{R}=\mathrm{H}$ or $\mathrm{OH}$

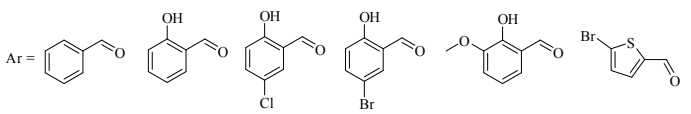

Scheme 1: Synthetic pathway for compounds PKB 1-10. (a) L-Proline (30 mol\%), DMSO, $80^{\circ} \mathrm{C}, 8 \mathrm{~h}$; (b) TFA, CH3NO2, reflux , 4-7 $\mathrm{h}$

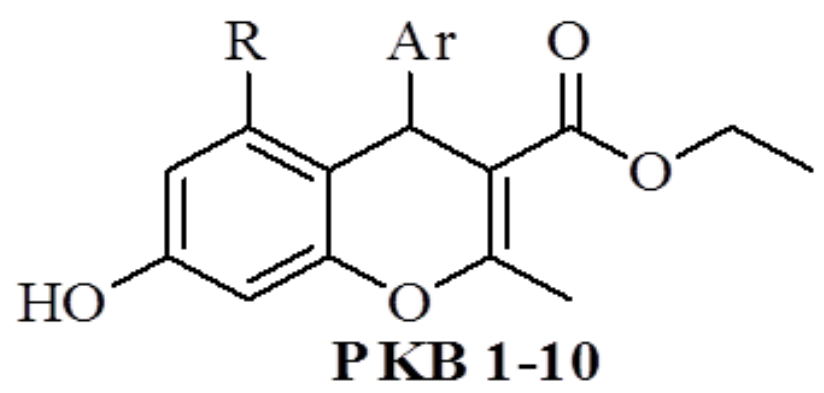

\begin{tabular}{|c|c|c|c|}
\hline Compounds & $\mathrm{GI}_{50}(\mu \mathrm{g} / \mathrm{ml})^{\mathrm{a}}$ & Compounds & $\mathrm{GI}_{50}(\mu \mathrm{g} / \mathrm{ml})^{\mathrm{a}}$ \\
\hline PKB-1 & 65.20 & PKB-7 & $3.72 \times 10^{3}$ \\
\hline PKB-2 & 7.20 & PKB-8 & 15.04 \\
\hline PKB-3 & 40.40 & PKB-9 & 21.06 \\
\hline PKB-4 & 2.37 & PKB-10 & 4.11 \\
\hline PKB-5 & 3.26 & Adriamycin & $<10$ \\
\hline PKB-6 & 34.20 & & \\
\hline
\end{tabular}

${ }^{\mathrm{a}} \mathrm{GI}{ }_{50}=$ Concentration of drug causing $50 \%$ inhibition of cell growth

\begin{tabular}{|c|c|c|c|c|c|c|c|}
\hline \multirow{2}{*}{ Compounds } & \multicolumn{2}{|c|}{ Toxicity Risks } & \multicolumn{5}{c|}{ Osiris Calculations } \\
\cline { 2 - 8 } & MUT & TUMO & MW & CLP & S & DL & D-S \\
\hline & Green & Green & 326 & 2.88 & -3.32 & -6.46 & 0.41 \\
\hline PKB-1 & Green & Green & 360 & 3.49 & -4.05 & -5.73 & 0.36 \\
\hline PKB-2 & Green & Green & 404 & 3.60 & -4.15 & -8.04 & 0.34 \\
\hline PKB-3 & Green & Green & 395 & 4.02 & -4.47 & -8.37 & 0.31 \\
\hline PKB-4 & Green & Green & 326 & 2.88 & -3.32 & -4.43 & 0.42 \\
\hline PKB-6 & Green & Green & 342 & 2.53 & -3.02 & -5.08 & 0.42 \\
\hline PKB-7 & Red & Green & 372 & 2.46 & -3.04 & -6.11 & 0.20 \\
\hline PKB-8 & Green & Green & 376 & 3.14 & -3.76 & -4.24 & 0.38 \\
\hline PKB-9 & Green & Green & 421 & 3.26 & -3.85 & -6.54 & 0.35 \\
\hline PKB-10 & Green & Green & 411 & 3.68 & -4.18 & -6.99 & 0.33 \\
\hline
\end{tabular}


ester group was observed as a triplet at $\delta 1.30-1.07 \mathrm{ppm}$ and $>\mathrm{CH}_{2}$ of ester group was observed as multiplet at $\delta$ 4.49-3.96 ppm in all compounds. The single proton at $4^{\text {th }}$ position was observed as a singlet at around $\delta$ 4.16-5.31 ppm in all compounds. Three protons of the methyl group at $2^{\text {nd }}$ position were found as a singlet at around $\delta$ 2.43-1.87 ppm. Other aromatic and aliphatic protons were present at their respective place. In ${ }^{13} \mathrm{C}$ NMR of compound PKB-4 and PKB-10, $-\mathrm{CH}_{3}$ and $>\mathrm{CH}_{2}$ of ester group were observed at $\delta 60.7 \mathrm{ppm}$ and $14.3 \mathrm{ppm}$, respectively.

\section{In vitro Anticancer Screening}

The synthesized compounds (PKB 1-10) were tested for their in vitro anticancer activity against human cancer (MCF-7) cell line by using sulforhodamine B assay (SRB Assay). ${ }^{26}$ The $\mathrm{GI}_{50}$ concentration of all compounds was calculated with reference to a control sample. For each compound, $50 \%$ growth inhibition $\left(\mathrm{GI}_{50}\right)$ was determined from Sigmoidal dose-response curves and given in Table 4. For reference purposes, adriamycin (ADR) data were included.

The resultant data showed that compounds exhibited potent cytotoxic effects on human breast cancer MCF-7 cell line (Figure 4). Among the series, compound PKB-4 showed the most potent activity $\left(\mathrm{GI}_{50}=2.37\right.$ $\mu \mathrm{g} / \mathrm{ml})$. The compounds PKB-2 $\left(\mathrm{GI}_{50}=7.20 \mu \mathrm{g} / \mathrm{ml}\right)$, PKB-5 $\left(\mathrm{GI}_{50}=3.26 \mu \mathrm{g} / \mathrm{ml}\right)$ and PKB-10 $\left(\mathrm{GI}_{50}=4.11\right.$ $\mu \mathrm{g} / \mathrm{ml}$ ) also displayed significant activity as compared to standard, Adriamycin $\left(\mathrm{GI}_{50}<10 \mu \mathrm{g} / \mathrm{ml}\right)$. It had been found that the presence of 5-bromothiophen moiety accounted for potent activity as in compound PKB-4 and PKB-10. These compounds also displayed significant docking scores. The compound having no substitution at benzene ring attached to the benzopyran ring also exhibited potent activity. Compounds $\mathrm{PKB}-8$ $\left(\mathrm{GI}_{50}=15.04 \mu \mathrm{g} / \mathrm{ml}\right)$ and PKB-9 $\left(\mathrm{GI}_{50}=21.06 \mu \mathrm{g} / \mathrm{ml}\right)$ possessed promising activity. Other compounds also showed somewhat activity. The presence of methoxy moiety resulted in less activity as in compound PKB-7 $\left(\mathrm{GI}_{50}=3.72 \times 10^{3} \mu \mathrm{g} / \mathrm{ml}\right)$.

The results revealed that compounds (PKB 1-10) were active on the MCF-7 cell line; therefore, it can be postulated that compounds were active against ER-positive breast tumours. About $80 \%$ of breast cancers were reported as ER-positive.

The structures of synthesized compounds (PKB 1-10) were confirmed by ${ }^{1} \mathrm{H}-\mathrm{NMR},{ }^{13} \mathrm{C}-\mathrm{NMR}$ spectra, FT-IR and melting point analysis. The obtained data and melting points were matching with known data of literature. ${ }^{25}$

\section{Experimental Section}

\section{Molecular Docking}

The autodock tool (autodock 4.2) was used for creating PDBQT files from traditional PDB files. The sequence of WST (3AS,4R,9BR)-4-(4-Hydroxyphenyl)1,2,3,3a,4,9b-hexahydrocyclopenta[c]chromen-9-ol) was retrieved from UniProt database. ${ }^{27}$ The threedimensional structure of WST was downloaded from the PDB database. The drug compound structures were drawn using ACD chem sketch and converted into PDB format using an open Babel tool. The 3D structures of above targeted proteins were docked with designed compounds (PKB 1-10) using autodock software. The docking results were analyzed using the discovery studio visualize tool. The ligands were prepared in the autodock 4.2 for docking studies. The optimized ligands were docked into targeted proteins using "Ligand fit" model in autodock 4.2. The energy interaction between protein and ligand was calculated.

\begin{tabular}{|c|c|c|c|c|c|c|c|}
\hline \multicolumn{7}{|c|}{ Table 5: Compounds (PKB 1-10) differing in the substitution at R and Ar. } \\
\hline Compounds. ${ }^{\text {a,b }}$ & $\mathbf{R}$ & Ar & $\begin{array}{c}\text { Reaction } \\
\text { Time (hours) }\end{array}$ & Mol. formula & $\begin{array}{c}\text { Mol. } \\
\text { weight }\end{array}$ & $\begin{array}{c}\text { Yield } \\
(\%)^{\mathbf{c}}\end{array}$ & $\begin{array}{c}\text { MP } \\
\left({ }^{\circ} \mathbf{C}\right)\end{array}$ \\
\hline PKB-1 & $\mathrm{H}$ & 2-hydroxyphenyl & 5 & $\mathrm{C}_{19} \mathrm{H}_{18} \mathrm{O}_{5}$ & 326.34 & 71 & 151 \\
\hline PKB-2 & $\mathrm{H}$ & 5-chloro-2-hydroxyphenyl & 6 & $\mathrm{C}_{19} \mathrm{H}_{17} \mathrm{ClO}_{5}$ & 360.07 & 65 & 155 \\
\hline PKB-3 & $\mathrm{H}$ & 5-bromo-2-hydroxyphenyl & 6 & $\mathrm{C}_{19} \mathrm{H}_{17} \mathrm{BrO}_{5}$ & 404.23 & 64 & 170 \\
\hline PKB-4 & $\mathrm{H}$ & 5-bromothiophen & 6 & $\mathrm{C}_{17} \mathrm{H}_{15} \mathrm{BrO}_{4} \mathrm{~S}$ & 395.26 & 65 & 110 \\
\hline PKB-5 & $\mathrm{OH}$ & phenyl & 5 & $\mathrm{C}_{19} \mathrm{H}_{18} \mathrm{O}_{5}$ & 326.34 & 78 & 102 \\
\hline PKB-6 & $\mathrm{OH}$ & 2-hydroxyphenyl & 4 & $\mathrm{C}_{19} \mathrm{H}_{18} \mathrm{O}_{6}$ & 342.34 & 80 & 150 \\
\hline PKB-7 & $\mathrm{OH}$ & 2-hydroxy-5-methoxyphenyl & 5 & $\mathrm{C}_{20} \mathrm{H}_{20} \mathrm{O}_{7}$ & 372.36 & 73 & 120 \\
\hline PKB-8 & $\mathrm{OH}$ & 5-chloro-2-hydroxyphenyl & 4 & $\mathrm{C}_{19} \mathrm{H}_{17} \mathrm{ClO}_{6}$ & 376.78 & 75 & 162 \\
\hline PKB-9 & $\mathrm{OH}$ & 5-bromo-2-hydroxyphenyl & 4 & $\mathrm{C}_{19} \mathrm{H}_{17} \mathrm{BrO}_{6}$ & 421.23 & 76 & 174 \\
\hline PKB-10 & $\mathrm{OH}$ & 5-bromothiophen & 5 & $\mathrm{C}_{17} \mathrm{H}_{15} \mathrm{BrO}_{5} \mathrm{~S}$ & 411.26 & 75 & 130 \\
\hline
\end{tabular}

aReagents and conditions: 3 ( $1.2 \mathrm{mmol}), 4$ (1 mmol), TFA (1 mmol), nitromethane $(5 \mathrm{ml})$, reflux 4-7 h.

${ }^{\mathrm{b}}$ Final product was confirmed by the analysis of ${ }^{1} \mathrm{H} N M R,{ }^{13} \mathrm{C} N M R, \mathrm{FT}-\mathrm{IR}$ and melting points.

'Yield refers to pure products after chromatography using $20 \%$ ethyl acetate in hexane as eluent. 


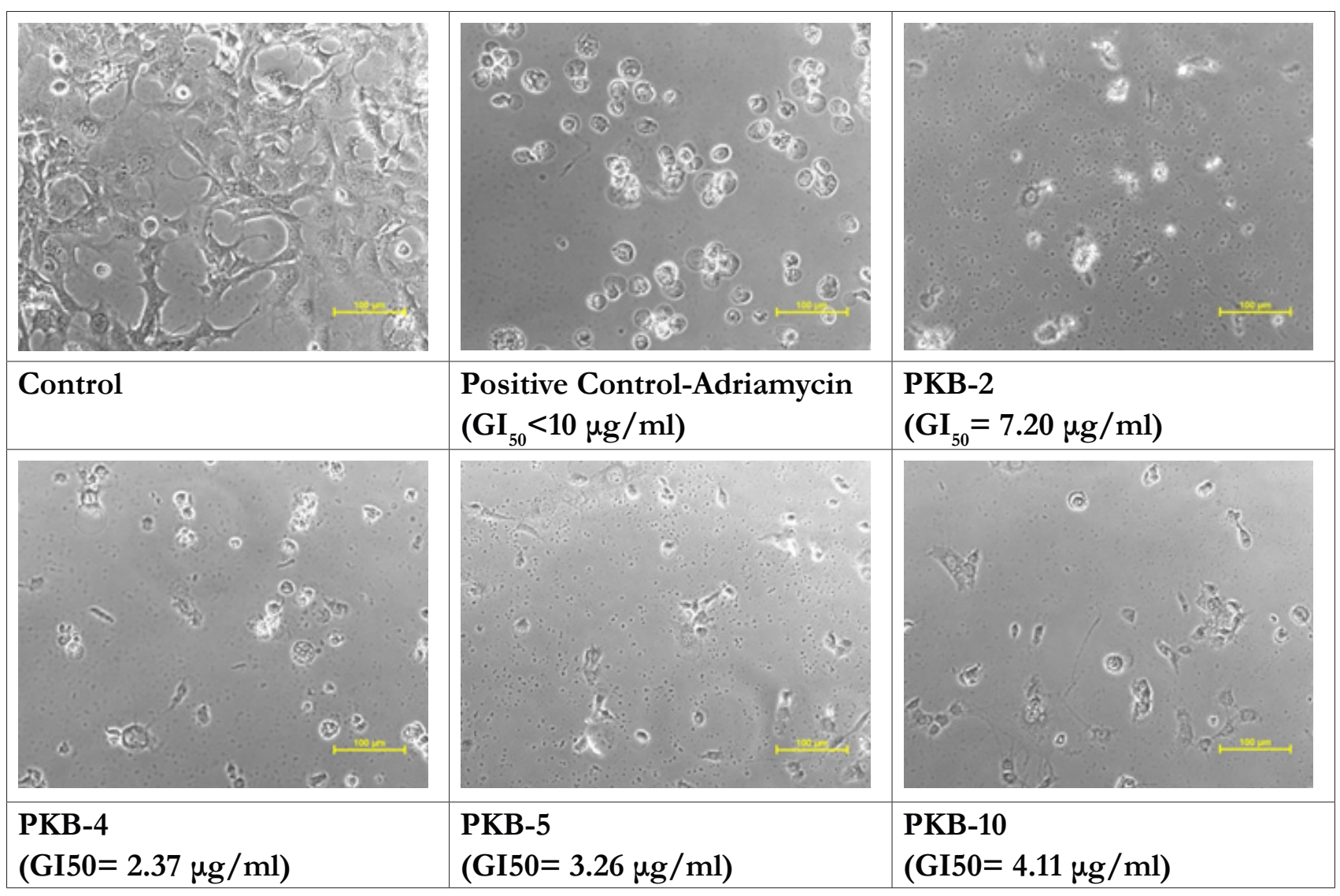

Figure 4: The inhibition growth $\left(\mathrm{Gl}_{50}\right)$ of MCF-7 cancer cells by PKB-2, PKB-4, PKB-5, PKB-10 and positive control (adriamycin).

\section{ADME Studies}

ADME properties of compounds (PKB 1-10) such as predicted gastrointestinal absorption, P-glycoprotein, blood-brain barrier and drug-likeness prediction such as Lipinski, Ghose and Veber rules and bioavailability score were predicted by online tool Swiss ADME of Swiss Institute of Bioinformatics (http://www.sib. swiss). ChemBioDraw Ultra version 15.0 (Cambridge Software) was used for drawing of $2 \mathrm{D}$ structural models and SMILES of each compound was translated into molfile by online Smiles translator and structure file generator found in Online tool Swiss ADME.

\section{Toxicity Studies}

Toxicity prediction studies were performed by Osiris Property Explorer. The Osiris Property Explorer includes the mol inspiration software through which the data may obtain. Mutagenic (MUT) and tumorigenic (TUMO) properties were predicted using Osiris molecular property explorer. Green colour predicts low toxicity; yellow shows moderate toxicity, while the red color predicts a high tendency for toxicity. Drug Score (D-S) of a compound predicts the compound's overall potential to qualify for a drug. It provides results based on molecular weight, cLogP, $\log \mathrm{S}$, drug- likeness and toxicity risks. Druglikeness (DL) values are based on topological descriptors, the fingerprint of molecular structure or other properties like molecular weight, solubility and cLogP. Molecular weight (MW) and aqueous solubility ( $\mathrm{S}$ ) were also predicted. The low aqueous solubility of a compound affects its absorption and distribution characteristics.

\section{Synthesis}

Physical and analytical data of synthesized derivatives were presented in Table 5 .

\section{Synthesis of substituted ethyl benzylidiene oxobutanoates (3)}

Intermediate compounds $\mathbf{3}$ were synthesized via known procedures. $^{25}$

Synthesis of substituted $4 \mathrm{H}-\mathrm{Chromene}$ derivatives (PKB 1-10)

The targeted final compounds (PKB 1-10) were synthesized via known procedures. ${ }^{25}$

Ethyl 7-hydroxy-4-(2-hydroxyphenyl)-2-methyl-4Hchromene-3-carboxylate (PKB-1): Yield: White solid (71\%); IR $\left(\mathrm{KBr}, \nu, \mathrm{cm}^{-1}\right): 3385(-\mathrm{OH}), 2986,2941,1742$, 1719, 1627, 1598, 1464; ${ }^{1} \mathrm{HNMR}\left(400 \mathrm{MHz}, \mathrm{CDCl}_{3}\right.$, ppm): 7.14 (d, J=8, 1H), 7.09-7.05 (m, 1H, Ar-H), 6.98 
(d, J=8, 1H), $6.85(\mathrm{~m}, 2 \mathrm{H}, \operatorname{Ar}-\mathrm{H}), 6.35(\mathrm{~s}, 1 \mathrm{H}), 6.32$ (s, 1H), 5.30 (bs, 1H, $-\mathrm{OH}), 4.20$ (s, 1H, >CH), 4.12-4.06 $\left(\mathrm{m}, 2 \mathrm{H},>\mathrm{CH}_{2}\right.$ ), 3.14 (bs, $\left.1 \mathrm{H},-\mathrm{OH}\right), 1.97$ (s, 3H, $-\mathrm{CH}_{3}$ ), $1.14\left(\mathrm{t}, \mathrm{J}=8,3 \mathrm{H},-\mathrm{CH}_{3}\right)$.

Ethyl 4-(5-chloro-2-hydroxyphenyl)-7-hydroxy-2methyl-4H-chromene-3-carboxylate (PKB-2): Yield: White solid (67\%); IR $\left(\mathrm{KBr}, \nu, \mathrm{cm}^{-1}\right): 3373(-\mathrm{OH}), 3001$, 2939, 1718, 1627, 1506, 1462, 1258; ${ }^{1} \mathrm{HNMR}(400 \mathrm{MHz}$, $\left.\mathrm{CDCl}_{3}, \mathrm{ppm}\right): 7.12$ (s, 1H), 7.04-6.97 (m, 2H), 6.77 (d, $J=8,1 \mathrm{H}), 6.37-6.34(\mathrm{~m}, 2 \mathrm{H}), 4.16(\mathrm{~s}, 1 \mathrm{H},>\mathrm{CH}-), 4.08(\mathrm{q}$, $\left.J=8,2 \mathrm{H},>\mathrm{CH}_{2}\right), 3.10$ (bs, $\left.1 \mathrm{H},-\mathrm{OH}\right), 1.96\left(\mathrm{~s}, 3 \mathrm{H},-\mathrm{CH}_{3}\right)$, $1.14\left(\mathrm{t}, \mathrm{J}=8,3 \mathrm{H},-\mathrm{CH}_{3}\right)$.

Ethyl 4-(5-bromo-2-hydroxyphenyl)-7-hydroxy-2methyl-4H-chromene-3-carboxylate (PKB-3): Yield: Red solid (66\%); IR $\left(\mathrm{KBr}, \nu, \mathrm{cm}^{-1}\right)$ : $3371(-\mathrm{OH}), 2982$, 2938, 1718, 1627, 1506, 1464, 1380, 1147; ${ }^{1} \mathrm{HNMR}$ (400MHz, $\left.\mathrm{CDCl}_{3}, \mathrm{ppm}\right): 7.26(\mathrm{~d}, 1 \mathrm{H}, J=4), 7.16(\mathrm{dd}, J=4$; $12,1 \mathrm{H}), 6.97(\mathrm{~d}, J=8,1 \mathrm{H}), 6.72(\mathrm{~d}, J=8,1 \mathrm{H}), 6.37-6.35$ $(\mathrm{m}, 2 \mathrm{H}), 4.16(\mathrm{~s}, 1 \mathrm{H},>\mathrm{CH}-), 4.11-4.06\left(\mathrm{~m}, 2 \mathrm{H},>\mathrm{CH}_{2}\right)$, 3.09 (bs, 1H, -OH), 1.96 (s, 3H, $\left.-\mathrm{CH}_{3}\right), 1.14$ (t, $\mathrm{J}=8,3 \mathrm{H}$, $\left.-\mathrm{CH}_{3}\right)$.

Ethyl 4-(5-bromothiophen-2-yl)-7-hydroxy-2methyl-4H-chromene-3-carboxylate (PKB-4): Yield: Brown solid (65\%); IR $\left(\mathrm{KBr}, v, \mathrm{~cm}^{-1}\right): 3426(-\mathrm{OH}), 2982$, 2899, 1690, 1629, 1507, 1459, 1261, 1146; ${ }^{1} \mathrm{HNMR}$ (400MHz, $\left.\mathrm{CDCl}_{3}, \mathrm{ppm}\right): 7.01(\mathrm{~d}, J=8,1 \mathrm{H}), 6.76(\mathrm{~d}, J=4$; $1 \mathrm{H}$, thiophenyl), 6.57 (dd, $J=2.5 ; 8,1 \mathrm{H}), 6.52(\mathrm{~s}, 1 \mathrm{H})$, 6.45 (d, J=4, 1H, thiophenyl), 5.18 (s,1H, >CH-), 4.23$4.16\left(\mathrm{~m}, 2 \mathrm{H},>\mathrm{CH}_{2}\right), 2.43\left(\mathrm{~s}, 3 \mathrm{H},-\mathrm{CH}_{3}\right), 1.28(\mathrm{t}, \mathrm{J}=8,3 \mathrm{H}$, $\left.-\mathrm{CH}_{3}\right) ;{ }^{13} \mathrm{C} \mathrm{NMR}\left(200 \mathrm{MHz}, \mathrm{CDCl}_{3}\right): 166.9,161.1,155.6$, 152.4, 150.4, 129.9, 129.3, 124.1, 115.8, 112.5, 110.6, 105.8, 103.2, 60.7, 36.1, 19.7, 14.3.

Ethyl 5,7-dihydroxy-2-methyl-4-phenyl-4Hchromene-3-carboxylate (PKB-5): Yield: White solid (76\%); IR (KBr, v, $\left.\mathrm{cm}^{-1}\right): 3446(-\mathrm{OH}), 3350(-\mathrm{OH}), 2987$, 2909, 1662, 1624, 1519, 1336,1127; ${ }^{1} \mathrm{HNMR}(400 \mathrm{MHz}$, $\left.\mathrm{CDCl}_{3}, \mathrm{ppm}\right)$ : 7.29-7.22 (m, 3H), 7.22-7.13 (m, 2H), 6.17 $(\mathrm{d}, J=2.3,1 \mathrm{H}), 6.01(\mathrm{~d}, J=2.3,1 \mathrm{H}), 5.01(\mathrm{~s}, 1 \mathrm{H},>\mathrm{CH}-)$, 4.13-4.07 (m, 2H, $\left.>\mathrm{CH}_{2}\right), 2.43\left(\mathrm{~s}, 3 \mathrm{H},-\mathrm{CH}_{3}\right), 2.10$ (bs, $1 \mathrm{H},-\mathrm{OH}), 1.22\left(\mathrm{t}, \mathrm{J}=8,3 \mathrm{H},-\mathrm{CH}_{3}\right)$.

Ethyl 5,7-dihydroxy-4-(2-hydroxyphenyl)-2-methyl4H-chromene-3-carboxylate (PKB-6): Yield: White solid (77\%); IR ( $\left.\mathrm{KBr}, \nu, \mathrm{cm}^{-1}\right): 3339(-\mathrm{OH}), 2985,2949$, 1717, 1632, 1513, 1485, 1381, 1145; ${ }^{1} \mathrm{HNMR}(400 \mathrm{MHz}$, DMSO-d ${ }^{6}, \mathrm{ppm}$ ): 9.50 (bs, 1H, $\left.-\mathrm{OH}\right), 9.09$ (bs, 1H, $-\mathrm{OH}), 7.27(\mathrm{~d}, J=8,1 \mathrm{H}), 7.06(\mathrm{t}, J=8,1 \mathrm{H}), 6.84(\mathrm{t}, J=8$, $1 \mathrm{H}), 6.78(\mathrm{~d}, J=8,1 \mathrm{H}), 5.85(\mathrm{~s}, 1 \mathrm{H},>\mathrm{CH}-), 5.67(\mathrm{~s}, 1 \mathrm{H})$, 4.50 (s, 1H), 4.09-3.97 (m, 2H), 3.28 (bs, 1H, -OH), 1.87 (s, 3H), 1.08 (t, $\left.J=8,3 \mathrm{H},-\mathrm{CH}_{3}\right) ;{ }^{13} \mathrm{C}$ NMR $(200 \mathrm{MHz}$, DMSO-d $\left.\mathrm{d}^{6}\right): 169.2,157.1,155.4,152.9,151.4,128.2$, 127.8, 127.5, 121.4, 115.9, 103.1, 98.1, 96.3, 94.4, 61.1, 30.1, 25.9, 14.3.
Ethyl 5,7-dihydroxy-4-(2-hydroxy-5methoxyphenyl)-2-methyl-4H-chromene-3carboxylate (PKB-7): Yield: White solid (73\%); IR $\left(\mathrm{KBr}, \nu, \mathrm{cm}^{-1}\right): 3443(-\mathrm{OH}), 2982,2943,1743,1726$, 1623, 1513; ${ }^{1} \mathrm{HNMR}$ (400MHz, DMSO-d ${ }^{6}$, ppm): 9.47 (bs, 1H, - $\mathrm{OH}), 9.08$ (bs, 1H, $-\mathrm{OH}), 6.88-6.86(\mathrm{~m}, 1 \mathrm{H})$, 6.80-6.77 (m, 2H), 5.84 (s, 1H, >CH-), 5.67 (s, 1H), 4.50 $(\mathrm{s}, 1 \mathrm{H}), 4.49-3.96(\mathrm{~m}, 2 \mathrm{H}), 3.71\left(\mathrm{~s}, 3 \mathrm{H},-\mathrm{OCH}_{3}\right), 3.26$ (bs, $1 \mathrm{H},-\mathrm{OH}), 1.87(\mathrm{~s}, 3 \mathrm{H}), 1.07\left(\mathrm{t}, J=8,3 \mathrm{H},-\mathrm{CH}_{3}\right) ;{ }^{13} \mathrm{C}$ NMR (200 MHz, DMSO-d $\left.\mathrm{d}^{6}\right)$ 169.2, 157.1, 155.4, 153.0, 147.5, 140.5, 128.4, 121.3, 119.3, 110.9, 103.0, 97.9, 96.3, 94.4, 61.1, 55.9, 43.6, 30.09, 25.9, 14.3.

Ethyl 4-(5-chloro-2-hydroxyphenyl)-5,7-dihydroxy2-methyl-4H-chromene-3-carboxylate (PKB-8): Yield: Red solid (74\%); IR ( $\left.\mathrm{KBr}, \nu, \mathrm{cm}^{-1}\right): 3398(-\mathrm{OH})$, 2994, 1714, 1626, 1613, 1512, 1479, 1374, 1149; ${ }^{1} \mathrm{HNMR}$ $\left(400 \mathrm{MHz}, \mathrm{CDCl}_{3}, \mathrm{ppm}\right): 7.31(\mathrm{~d}, J=4,1 \mathrm{H}), 6.99$ (dd, $J=$ 4, 8, 1H), $6.74(\mathrm{~d}, J=8,1 \mathrm{H}), 5.95(\mathrm{~s}, 1 \mathrm{H}), 5.85(\mathrm{~s}, 1 \mathrm{H})$, 4.57 (s, 1H, >CH-), 4.14-4.08 (m, 2H), 3.05 (bs, $1 \mathrm{H}$, $-\mathrm{OH}), 2.59$ (bs, $1 \mathrm{H},-\mathrm{OH}), 1.93$ (s, 3H, $\left.-\mathrm{CH}_{3}\right), 1.17$ (t, $\left.J=8,3 \mathrm{H},-\mathrm{CH}_{3}\right)$.

Ethyl 4-(5-bromo-2-hydroxyphenyl)-5,7-dihydroxy-2methyl-4H-chromene-3-carboxylate (PKB-9): Yield: Red solid (74\%); IR $\left(\mathrm{KBr}, \nu, \mathrm{cm}^{-1}\right)$ : $3404(-\mathrm{OH}), 2925$, 1714, 1624, 1512, 1477, 1374, 1249; ${ }^{1} \mathrm{HNMR}(400 \mathrm{MHz}$, $\left.\mathrm{CDCl}_{3}, \mathrm{ppm}\right): 7.46(\mathrm{~d}, J=2,1 \mathrm{H}), 7.15(\mathrm{dd}, J=4,8,1 \mathrm{H})$, $6.69(\mathrm{~d}, J=8,1 \mathrm{H}), 5.96(\mathrm{~d}, J=4,1 \mathrm{H}), 5.83(\mathrm{~d}, J=4,1 \mathrm{H})$, 4.57 (s, 1H, >CH-), 4.14-4.09 (m, 2H), 3.05 (bs, 1H, $-\mathrm{OH}), 2.57$ (bs, 1H, -OH), 1.93 (s, 3H,-CH $), 1.17$ (t, $\left.J=8,3 \mathrm{H},-\mathrm{CH}_{3}\right)$.

Ethyl 4-(5-bromothiophen-2-yl)-5,7-dihydroxy2-methyl-4H-chromene-3-carboxylate (PKB-10): Yield: Off white solid (75\%); IR (KBr, v, $\left.\mathrm{cm}^{-1}\right)$ : 3512 (-OH), $3333(-\mathrm{OH}), 2980,1695,1528,1494,1375,1211$; ${ }^{1} \mathrm{HNMR}\left(400 \mathrm{MHz}, \mathrm{CDCl}_{3}, \mathrm{ppm}\right): 6.76(\mathrm{~d}, J=4,1 \mathrm{H}), 6.54$ (d, $J=4 ; 1 \mathrm{H}$, thiophenyl), $6.15(\mathrm{~d}, J=4,1 \mathrm{H}), 6.09(\mathrm{~d}, J=4$, $1 \mathrm{H}$, thiophenyl), 5.32 (s, 1H, >CH-), 4.24-4.19 (m, $2 \mathrm{H}$, $\left.>\mathrm{CH}_{2}\right), 2.41\left(\mathrm{~s}, 3 \mathrm{H},-\mathrm{CH}_{3}\right), 1.30\left(\mathrm{t}, \mathrm{J}=8,3 \mathrm{H},-\mathrm{CH}_{3}\right) ;{ }^{13} \mathrm{C}$ NMR $\left(200 \mathrm{MHz}, \mathrm{CDCl}_{3}\right): 166.9,161.2,156.0,153.9$, 151.5, 150.8, 129.2, 124.7, 110.7, 106.1, 104.4, 99.5, 96.1, 60.7, 31.3, 19.7, 14.3 .

\section{In vitro Anticancer Activity by Sulforhodamine B Assay}

The in vitro cytotoxic activity was checked by using Sulforhodamine B staining (SRB) assay. ${ }^{26}$ The cell lines were grown in RPMI 1640 medium containing 10\% fetal bovine serum and $2 \mathrm{mM} \mathrm{L-glutamine.} \mathrm{Cells} \mathrm{were} \mathrm{plated}$ in 96 multiwell plates (104 cell/ well) and plates were incubated at $37^{\circ} \mathrm{C}, 5 \% \mathrm{CO}_{2}, 95 \%$ air and $100 \%$ relative humidity for $24 \mathrm{~h}$ prior to addition of experimental compounds. Experimental compounds were initially 
solubilized in dimethyl sulfoxide and prepared different molar concentrations. Different molar concentrations of the test compounds $\left(10^{-7}, 10^{-6}, 10^{-5}\right.$ and $\left.10^{-4} \mathrm{M}\right)$ were put into the cell line monolayer. For each individual dose, triplicate wells were made. Test compounds were incubated into monolayer cells for almost $48 \mathrm{hr}$ at $37^{\circ} \mathrm{C}$ and in an atmosphere of $5 \% \mathrm{CO}_{2}$. Then after completion of $48 \mathrm{hr}$, cells were fixed, washed and stained with Sulforhodamine B stain. If there made the excess stain, it would be washed with acetic acid. The plates were air dried. Bound stain was subsequently eluted with $10 \mathrm{mM}$ trizma base and the absorbance was read on an Elisa plate reader at a wavelength of $540 \mathrm{~nm}$ with $690 \mathrm{~nm}$ reference wavelength. The relation between surviving fraction and different drug concentration was plotted and $\mathrm{GI}_{50}$ was calculated for each compound.

\section{CONCLUSION}

It could be concluded that most of the compounds in series PKB 1-10 exhibited potent anticancer activity on MCF-7 cell line. Compound PKB-4 $\left(\mathrm{GI}_{50}=2.37\right.$ $\mu \mathrm{g} / \mathrm{ml}$ ) possessed most potent activity as compared to standard, Adriamycin $\left(\mathrm{GI}_{50}<10 \mu \mathrm{g} / \mathrm{ml}\right)$. Docking, in silico ADME and toxicity studies were also favorable for the synthesis of compounds. The presence of 5-bromothiophen moiety (PKB-4 and PKB-10) was favorable for binding as well as anti-breast cancer activity. All compounds (PKB 1-10) were found to be active on the MCF-7 cell line, therefore, compounds might be active against ER-positive breast cancer. Further evaluation of the detail mechanism pathway involved in an activity needs to be investigated

\section{ACKNOWLEDGEMENT}

The authors thankful to the Advanced Centre for Treatment Research and Education in Cancer (ACTREC), Navi Mumbai, Maharashtra, India, for evaluation of the in vitro anticancer activity.

\section{CONFLICT OF INTEREST}

The authors declare no conflict of interest.

\section{ABBREVIATIONS}

ADME: Absorption, Distribution, Metabolism, Excretion; GI: Gastro Intestinal; P-gp: P-glycoprotein; BBB: Blood Brain Barrier; CYP1A2: Cytochrome P450 family 1 subfamily A member 2; CYP2D6: Cytochrome P450 family 2 subfamily D member 6; FT-IR: Fouriertransform infrared spectroscopy; ${ }^{1} \mathbf{H}-\mathbf{N M R}$ : Proton nuclear magnetic resonance; ${ }^{13}$ C-NMR: Carbon-13 nuclear magnetic resonance; GABA: Gamma aminobutyric acid; TMS: Tertramethyl Silane; TLC: Thin layer chromatography; WST: ((3AS,4R,9BR)-4-(4Hydroxyphenyl)-1,2,3,3a,4,9b-hexahydrocyclopenta[c] chromen-9-ol).

\section{REFERENCES}

1. Bray F, Ferlay J, Soerjomataram I, Siegel RL, Torre LA, Jemal A, et al. Global cancer statistics 2018: Globocan estimates of incidence and mortality worldwide for 36 cancers in 185 countries. CA Cancer J Clin. 2018;68(6):3944.

2. https://www.who.int/cancer/prevention/diagnosis-screening/breast-cancer/ en/

3. Mesquita MLD, Araújo RM, Bezerra DP, Filho RB, Paula JED, Silveira ER, et al. Cytotoxicity of $\delta$-tocotrienols from Kielmeyera coriacea against cancer cell lines. Bioorg Med Chem. 2011;19(1):623-30.

4. Noushini1 S, Alipour1 E, Emami S, Safavi M, Ardestani SK, Gohari AR, et al. Synthesis and cytotoxic properties of novel (E)-3-benzylidene-7methoxychroman-4-one Derivatives. DARU J Pharmaceut Sci. 2013;21(1):31.

5. Colombano G, Travelli C, Galli U, Caldarelli A, Chini MG, Canonico PL, et al. A novel potent nicotinamide phosphoribosyl transferase inhibitor synthesized via click chemistry. J Med Chem. 2010;53(2):616-23.

6. Costa M, Dias TA, Brito A, Proença F. Biological importance of structurally diversified chromenes. Eur J Med Chem. 2016;123(10):487-507.

7. Puppala M, Zhao X, Casemore D, Zhou B, Aridoss G, Narayanapillai S, et al. 4H-chromene-based anticancer agents towards multi-drug resistant $\mathrm{HL60} /$ MX2 human leukemia: SAR at the $4^{\text {th }}$ and $6^{\text {th }}$ positions. Bioorg Med Chem. 2016;24(6):1292-7.

8. Rehse K, Schinkel W. Anticoagulante 3-aralkyl-4- hydroxy-2-pyrone. Arch Pharm. 1983;316(12):988-94.

9. Shah NK, Shah NM, Patel MP, Patel RG. Synthesis of 2-amino-4H-chromene derivatives under microwave irradiation and their antimicrobial activity. J Chem Sci 2013;125(3):525-30.

10. Fatima I, Saxena R, Kharkwal G, Hussain MK, Yadav N, Hajela K, et al. The anti-proliferative effect of 2- [piperidinoethoxyphenyl]-3-[4-hydroxyphenyl]$2 \mathrm{hbenzo}(\mathrm{b})$ pyran is potentiated via induction of estrogen receptor beta and p21 in human endometrial adenocarcinoma cells. J Steroid Biochem Mol Biol. 2013;138:123-31.

11. Gregor W, Grabner G, Adelwohrer C, Rosenau T, Gille L. Antioxidant Properties of Natural and Synthetic Chromanol Derivatives: Study by fast kinetics and electron spin resonance spectroscopy. J Org Chem. 2005;70(9):3472-83.

12. Boukattaya F, Daoud A, Boeda F, Long MSMP, Gharsallah N, Kadri A, et al. Synthesis and biological evaluation of 3-cyano-4h-chromene derivatives bearing carbamate functionality. Med Chem. 2019;15(3):257-64.

13. Carr M, Knox AJS, Nevin DK, Boyle NO, Wang S, Egan B, et al. Optimization of estrogen receptor subtype-selectivity of a 4-aryl-4h-chromene scaffold previously identified by virtual screening. Bioorg Med Chem. 2020;28(5):115261.

14. Conti C, Monaco LP, Desideri N. Synthesis and anti-rhinovirus activity of novel 3-[2-(pyridinyl)vinyl] substituted -2H-chromenes and $-4 \mathrm{H}$-chromen-4ones. Bioorg Med Chem. 2014;22(3):1201-7.

15. Nimesh RK, Dhaval DH, Prashant T, Saurabh KP. Synthesis and evaluation of in vitro antitubercular activity and antimicrobial activity of some novel 4H-chromeno[2,3-d]pyrimidine via 2- amino-4-phenyl-4H-chromene-3carbonitriles. Med Chem Res. 2011;20(7):854-64.

16. Nitin K, Sushil K, Himanshu G, Sharma PK. 3-Hydroxy-2- (substituted phenyl) -4H-chromen-4-one derivatives- synthesis, spectral characterization and pharmacological screening. World Res J Biochem. 2012;1(1):1-5.

17. Chauhan D, Hashim SR, Rani P, Kumar S, Shrimal N, Shastri D, et al. Discovery of novel substituted $\mathrm{N}$-(6-chloro-3-cyano-4-phenyl-4Hchromen-2-yl)-2-(4-chloro-phenoxy)-acetamide for biphasic anticancer and anticonvulsant activities. Med Chem. 2019;15:1. 
18. Comşa S, Cîmpean AM, Raica M. The story of MCF-7 breast cancer cell line: 40 years of experience in research. Anticancer Res. 2015;35(6):3147-54.

19. Zanger UM, Schwab M. Cytochrome P450 enzymes in drug metabolism: Regulation of gene expression, enzyme activities and impact of genetic variation. Pharmacol Therapeut. 2013;138(1):103-41.

20. Nanayakkara AK, Follit CA, Chen G, et al. Targeted inhibitors of P-glycoprotein increase chemotherapeutic-induced mortality of multidrug resistant tumor cells. Sci Rep. 2018;8(1):967.

21. Alrushaid S, Sayre CL, Yáñez JA, Forrest ML, Senadheera SN, Burczynski FJ, et al. Pharmacokinetic and toxicodynamic characterization of a novel doxorubicin derivative. Pharmaceutics. 2017;9(3):35.

22. Sardi I, Marca GL, Cardellicchio S, Giunti L, Malvagia S, Genitori L, et al. Pharmacological modulation of blood-brain barrier increases permeability of doxorubicin into the rat brain. Am J Cancer Res. 2013;3(4):424-32.

23. Ohnishi T, Tamai I, Sakanaka K, Sakata A, Yamashima T, Yamashita J, et al. In vivo and in vitro evidence for ATP-dependency of P-glycoprotein- mediated efflux of doxorubicin at the blood-brain barrier. Biochem Pharmacol. 1995;49(10):1541-4.

24. Kivisto KT, Kroemer HK, Eichelbaum M. The role of human cytochrome P450 enzymes in the metabolism of anticancer agents: Implications for drug interactions. Br J Clin Pharmacol. 1995;40(6):523-30.

25. Priyanka, Sharma RK, Butcher RJ, Katiyar D. Facile construction of $4 \mathrm{H}$-chromenes via michael addition of phenols to benzylidene oxobutanoates and their successful conversion into pyranocoumarins. Tetrahedron Lett. 2018;59(24):2347-51.

26. Skehan P, Storeng R, Scudiero D, Monks A, McMahon J, Vistica D, et al. New colorimetric cytotoxicity assay for anticancer-drug screening. J Natl Cancer Inst. 1990;82(13):1107-12.

27. Richardson TI, Norman BH, Lugar CW, Jones SA, Wang Y, Durbin JD, et al. Benzopyrans as selective estrogen receptor beta agonists (SERBAs). part 2: Structure activity relationship studies on the benzopyran scaffold. Bioorg Med Chem Lett. 2007;17(13):3570-74.

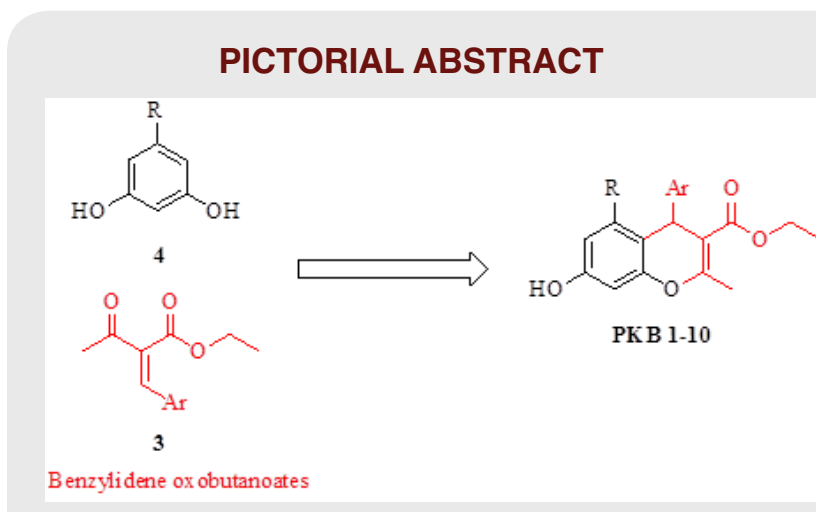

\section{SUMMARY}

The $4 \mathrm{H}$-chromenes are important chemical synthon, associated with a broad range of biological activities, including anticancer activity. The computational studies like docking, ADME and toxicity were performed and based on their results, compounds were designed and synthesized. The anticancer study of $4 \mathrm{H}$-chromene derivatives (PKB 1-10) was carried out by Sulforhodamine B stain (SRB) assay on MCF7 cell line. The compounds PKB-2, PKB-4, PKB-5 and PKB-10 displayed similar activity $\left(\mathrm{GI}_{50}=<10 \mu \mathrm{g} / \mathrm{ml}\right)$ as standard drug, Adriamycin. Most of the compounds displayed significant activity.

\section{About Authors}

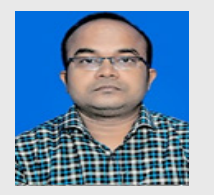

Mr. Piyush Kumar is pursuing his Ph.D. from Integral University, Lucknow, India. He has done MS Pharm from NIPER, Raebareli, India. He had worked as a Research Associate and Project Scientist in the Department of Chemistry, IIT Kanpur. His research interest in the area of organic chemistry, medicinal chemistry and nanotechnology.

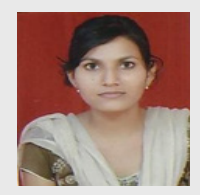

Dr. Pinki Rawat is currently working as Associate Professor at Maharana Pratap College of Pharmacy, Kanpur, Uttar Pradesh, India. She has completed her Ph.D. from Birla Institute of Technology, Ranchi, India. Her research interest is in the area of synthesis of heterocyclic compounds, molecular docking, QSAR, antiepileptic, anticancer, antimicrobial and antioxidant.

Cite this article: Kumar P, Rawat P. Docking Studies, Synthesis and Evaluation of Anticancer Activity of 4H-Chromene Derivatives. Indian J of Pharmaceutical Education and Research. 2021;55(1):256-65. 